\title{
Cenário da reabilitação cardíaca no centro do estado do Rio Grande do Sul
}

\author{
Cardiac rehabilitation scenario in the state \\ of Rio Grande do Sul, Brazil
}

\author{
Leticia Castagna, ${ }^{1}$ Tiago José Nardi Gomes² \\ 'Centro Universitário Franciscano, Santa Maria, RS, Brasil. \\ 2 Instituto de Cardiologia, Porto Alegre, RS, Brasil.
}

Recebido em: 25/05/2017 / Aceito em: 07/07/2017 / Publicado em: 01/10/2017

leticiacastagnaa@gmail.com

\section{RESUMO}

Objetivo: identificar os programas de reabilitação cardíaca existentes em Santa Maria, na região central do Rio Grande do Sul e compará-los a um serviço de um hospital referência e observar se as condutas estão de acordo com a Diretriz da Sociedade Brasileira de Cardiologia vigente. Método: estudo exploratório, analítico descritivo e comparativo, do tipo levantamento epidemiológico de caráter quantitativo. No período de agosto a outubro de 2015. Resultados: a predominância do sexo masculino e da variação de 42 a 78 anos de idade. Os serviços $\mathrm{A}$ e $\mathrm{C}$ obtiveram maior índice em relação a IAM e ICC, ao contrário de $\mathrm{C}$ em que prevaleceu a CRM e as demais doenças cardiovasculares. Considerações finais: os serviços de reabilitação cardíaca de Santa Maria versus um hospital de referência estão no mesmo nível de qualidade perante as recomendações descritas na última literatura vigente, sendo um ponto positivo para uma cidade de médio porte como Santa Maria.

Palavras-chave: Centros de Reabilitação; Doenças cardiovasculares; Exercício físico e Reabilitação.

\section{ABSTRACT}

Objective: identify cardiac rehabilitation programs in Santa Maria in the central region of Rio Grande do Sul, and compare them to a service of a reference hospital observing if the conducts are in agreement with the current guidelines of the Brazilian Society of Cardiology. Method: exploratory, descriptive analytical and comparative study of the type epidemiological survey with quantitative disposition. Results: within subjects we found the predominance of male in the age range of 42-78 years old. Services $A$ and $C$ had a higher ratio in the acute myocardial infarction (AMI) and congestive heart failure (CHF) unlike $C$ that prevailed myocardial revascularization surgery (MRC) and other cardiovascular diseases. Closing remarks: the cardiac rehabilitation services at Santa Maria versus a reference hospital are in the same level of quality towards the recommendations described in the current literature, being a positive point for a midsize city as Santa Maria.

Keywords: Rehabilitation Centers; Cardiovascular diseases; Physical exercises and Rehabilitation.

\section{INTRODUÇÃO}

A morbimortalidade cardiovascular é uma das maiores pandemias nos cenários da área da saúde atual. O Ministério da Saúde (MS) realizou uma pesquisa da ocorrência desse diagnóstico, no ano de 2010, sendo observadas 326 mil mortes por doenças cardiovasculares (DCVs); destes 200 mil devido a doenças isquêmicas do coração e de DCVs, havendo um descontrole no cenário atual. ${ }^{1}$ Segundo dados do Departamento de Informática do Sistema Único de Saúde (DATASUS) em Santa Maria - RS, no ano de 2013 ocorreram 101 mortes relacionadas ao infarto agudo do miocárdio (IAM), 40 mortes de insuficiência cardíaca (IC) e 19 mortes de indivíduos com cardiomiopatias, sendo as faixas etárias mais acometidas dos 60 aos 80 anos. $^{2}$

Após a etapa de recuperação do paciente ter um evento que trouxe dano ao sistema cardiovascular, a reabilitação cardíaca ( $\mathrm{RC}$ ) torna-se necessária. Este processo é uma combinação de atividades e exercícios que proporcionam aos indivíduos cardiopatas as melhores condições físicas, mentais e sociais, fazendo com que 
possam realizar a manutenção e a sua retomada as suas atividades profissionais e familiares por conta própria. ${ }^{3-6}$

O exercício físico tem sido um grande aliado dos programas de reabilitação cardíaca (PRC), com objetivo de prevenção e reabilitação que ajudam a promover uma melhora da aptidão física e da capacidade funcional. ${ }^{7,8}$ As práticas destes protocolos de exercícios voltados a indivíduos cardiopatas podem trazer benefícios cardiorrespiratórios, motores e psicossociais, portanto, a relevância e a forma de intensidade da resposta cardiovascular irão provir das particularidades de um exercício executado, ou seja, a intensidade, a duração e a massa muscular envolvida. 9,10

Em consonância com o aumento na incidência das DCVs, os PRC vêm crescendo significativamente em todo Brasil. No entanto, não existem informações específicas dos perfis dos pacientes e métodos utilizados nestes centros, portanto a pertinência deste estudo se deve à captação mais precisa destas informações. Desta forma, este estudo objetivou investigar os programas de reabilitação cardíaca existentes na região central do Rio Grande do Sul e compará-los a um serviço de RC de um hospital referência. Após foi observado se os mesmos estão em conformidade com as recomendações da última diretriz vigente de RC.

\section{MÉTODO}

Estudo exploratório, analítico e/ou descritivo e comparativo, do tipo levantamento epidemiológico. Esta pesquisa foi realizada em três centros de reabilitação cardíaca do município de Santa Maria - RS e no Instituto de Cardiologia do Rio Grande do Sul. A coleta de dados ocorreu nos meses de agosto a outubro de 2015 via visita física no local, em contato direto com o responsável. Para preservar a identidade de cada serviço de reabilitação, quando os mesmos forem citados no decorrer desse manuscrito estarão nomeados pelas letras $A, B, C$ e $D$.

A amostra foi composta de todos os locais que se enquadraram nos seguintes critérios de inclusão: serviços de RC que funcionassem a mais de um ano com local físico, contendo cardiologista ou técnico superior responsável pelo serviço, localizados na região central do RS, assinatura do termo de autorização da pesquisa pelo responsável técnico do local de pesquisa, assinatura do termo de consentimento livre e esclarecido. Os critérios de exclusão deste estudo foram os questionários incompletos e os serviços não quererem participar da pesquisa.

Para a coleta de dados foi utilizado um questionário de entrevista formulado pelos pesquisadores, contemplado por sete perguntas fechadas relacionadas às doenças cardiovasculares mais frequentes nos serviços, a frequência de atendimentos semanais, os protocolos fisioterapêuticos, os profissionais que compõem a equipe multiprofissional dos serviços, a faixa etária dos pacientes e o gênero mais prevalente.

Os dados coletados dos três serviços de reabilitação cardíaca da região central do município de Santa Maria - RS foram comparados a um hospital referência da América Latina. Posteriormente, observou-se se os mesmos estavam em conformidade com as recomendações da última diretriz vigente de reabilitação cardíaca, publicada pela Sociedade Brasileira de Cardiologia no ano de 2014, nomeada como Diretriz Sul-Americana de Prevenção e Reabilitação Cardiovascular.

Este estudo foi submetido ao Comitê de Ética e Pesquisa (CEP) do Centro Universitário Franciscano, seguindo o percurso e as normas éticas argumentadas na resolução 466/12, aprovado com $n^{\circ}$ do certificado de apresentação para apreciação ética (CAAE) 4912345.0.0000.5306.

\section{RESULTADOS}

Foram encontrados três serviços de reabilitação cardíaca na região central do Rio Grande do Sul. Um serviço vinculado à rede pública de saúde e dois a rede privada. Nestes três serviços pesquisados foram encontrados 123 pacientes, realizando reabilitação cardíaca nos últimos 2 anos. Na tabela abaixo, encontra-se a característica da amostra (Tabela 1). Em todos os serviços foi possível perceber a predominância do sexo masculino.

No gráfico 1, estão relacionadas às doenças cardiovasculares mais frequentemente encontradas nestes serviços de reabilitação cardíaca, sendo o motivo pelo qual os pacientes procuraram auxílio.

Tabela 1 - Levantamento epidemiológico acerca do gênero e de idades dos indivíduos participantes dos serviços de RC.

\begin{tabular}{lccc}
\hline Serviços & Faixa etária & Sexo Feminino & Sexo Masculino \\
\hline A & 42 a 70 anos $(n=48)$ & $30 \%(n=16)$ & $70 \%(n=32)$ \\
B & 44 a 78 anos $(n=65)$ & $30 \%(n=22)$ & $70 \%(n=43)$ \\
C & 55 a 78 anos $(n=10)$ & $20 \%(n=2)$ & $80 \%(n=8)$ \\
\hline
\end{tabular}

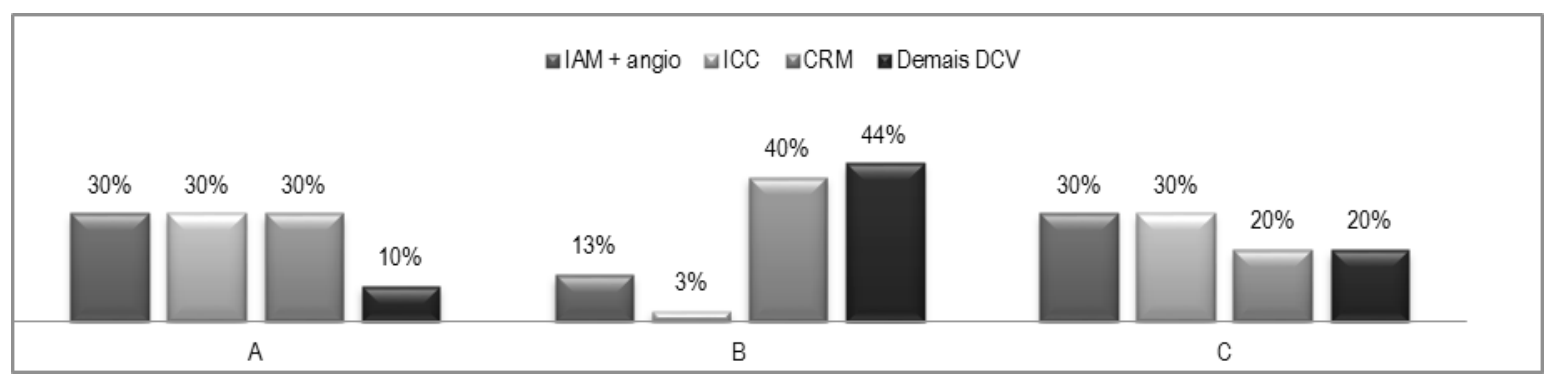

Tabela 1 - Doenças cardiovasculares mais frequentes encontradas nos serviços de reabilitação cardíaca A, B e C pesquisados. IAM - Infarto Agudo do Miocárdio; ICC - Insuficiência cardíaca congestiva; CRM - Cirurgia da Revascularização do Miocárdio. 
A tabela 2 refere-se aos protocolos dos programas de RC dos três serviços perante a avaliação inicial antes de iniciar o programa de RC na Fase II.

$\mathrm{Na}$ tabela 3 estão os dados abrangentes relacionados ao aquecimento. Os resultados encontrados foram semelhantes.

A próxima etapa, chamada de condicionamento, foi dividida em exercícios de fortalecimento e de resistência (Tabela 4).

O segundo item avaliado abrangeu o treinamento de resistência. Os serviços $A$ e $B$ utilizam o mesmo protocolo de exercícios, com caminhada na esteira em baixa velocidade ou bicicleta ergométrica com carga moderada, em um período de trinta minutos. $O$ serviço $C$ realizava exercícios rítmicos baseados em FC, durante um período pré-determinado e interrompidos conforme os pacientes se aproximam da FC alvo do treinamento de resistência. No serviço D, são utilizados exercícios para resistência cardiovascular na esteira/bike, durante trinta minutos com velocidade moderada e treinamento baseado na FC alvo.

O terceiro momento dos protocolos fisioterapêuticos em serviços de reabilitação cardíaca é o desaquecimento e o relaxamento. Na tabela 5 , estão os dados relacionados a este período, nos serviços $A, B, C$ e $D$ são realizados alongamentos de grandes grupos musculares de MMSS e MMII, em um período de dez a vinte minutos aproximadamente. O que diferenciou nesta etapa foi que, no serviço $C$, o relaxamento e o alongamento são realizados em meio aquático, favorecendo um melhor relaxamento dos pacientes.

Tabela 2 - Protocolos fisioterapêuticos dos programas de reabilitação cardíaca - avaliação inicial, frequência semanal, tempo de duração e aferição de sinais vitais iniciais e finais.

\begin{tabular}{|c|c|c|c|}
\hline Serviços & Avaliação inicial & $\begin{array}{c}\text { Frequência / } \\
\text { Tempo de Duração }\end{array}$ & $\begin{array}{l}\text { Aferição de sinais vitais } \\
\text { iniciais e finais }\end{array}$ \\
\hline A & $\begin{array}{l}\text { TC6', TD4, força de musculatura ventilatória, } \\
\text { exames de sangue e verificação de IMC }\end{array}$ & $2 \mathrm{x} / 2 \mathrm{~h}$ & $\begin{array}{c}\text { PA, Borg modificado, saturação, } \\
\text { FC, FR }\end{array}$ \\
\hline B & $\begin{array}{l}\text { TC6', TD4, força de musculatura ventilatória, } \\
\text { exames de sangue e verificação de IMC }\end{array}$ & $2 \mathrm{x} / 1 \mathrm{~h} 30 \mathrm{~min}$ & $\begin{array}{c}\text { PA, Borg modificado, saturação, } \\
\text { FC, FR }\end{array}$ \\
\hline C & $\begin{array}{l}\text { Espirometria, TC6 ', circunferência abdominal, } \\
\text { verificação de IMC e de força de musculatura } \\
\text { ventilatória, questionário de qualidade de Vida SF-36. }\end{array}$ & $2 \mathrm{x} / 1 \mathrm{~h}$ & PA, Borg, saturação, FC, FR \\
\hline D & $\begin{array}{l}\text { TC6', verificação de força da musculatura } \\
\text { ventilatória, testes de força muscular, FC repouso }\end{array}$ & $2 \mathrm{x} / 1 \mathrm{~h} 30$ & $\begin{array}{l}\text { PA, Borg modificado, FR, FC, } \\
\text { saturação }\end{array}$ \\
\hline
\end{tabular}

Legenda: TC6 - teste de caminhada de seis minutos; TD4 - teste do degrau de 4 minutos; PA - pressão arterial; FC - frequência cardíaca; FR - frequência respiratória.

Tabela 3 - Protocolos fisioterapêuticos dos programas de reabilitação cardíaca acerca do aquecimento.

\begin{tabular}{ll}
\hline Serviços & Aquecimento \\
\hline A & Exercícios sem carga, movimentos rítmicos e de média amplitude com duração de 10 a 12 min \\
B & Exercícios sem carga, movimentos rítmicos e com média amplitude com duração de 10 a 12 min \\
C & Exercícios sem carga, movimentos rítmicos e com média amplitude com duração de 10 min \\
D & Alongamentos de grandes grupos musculares de MMSS e MMII durante 10 min, sendo realizado aquecimento \\
& na bicicleta com velocidade leve durante 10 min \\
\hline
\end{tabular}

Legenda: MIMSS - membros superiores; MIMII - membros inferiores; min - minutos.

Tabela 4 - Protocolos fisioterapêuticos dos programas de reabilitação cardíaca acerca do condicionamento do paciente cardiopata, realizando exercícios de fortalecimento e resistência.

\begin{tabular}{|c|c|c|}
\hline \multirow[t]{2}{*}{ Serviços } & \multicolumn{2}{|l|}{ Condicionamento } \\
\hline & Fortalecimento & Resistência \\
\hline A & $\begin{array}{l}\text { Exercícios de fortalecimento de grandes grupos musculares de } \\
\text { MMII e MMSS de tronco ( } 2-3 \text { séries e } 8 \text { - } 10 \text { repetições) utilizando } \\
\text { baixa carga ou bandagens elásticas de média resistência com } \\
\text { duração de } 20 \text { min. Exercícios de musculatura ventilatória com o } \\
\text { aparelho Threshold com } 5 \text { séries e } 6 \text { repetições. }\end{array}$ & $\begin{array}{l}\text { Exercícios de resistência com caminhada na esteira } \\
\text { em baixa velocidade e bicicleta ergométrica de } \\
\text { carga moderada com duração de } 30 \text { min. }\end{array}$ \\
\hline$B$ & $\begin{array}{l}\text { Exercícios de fortalecimento de grandes grupos musculares } \\
\text { de MMSS/MMII e tronco com duração de } 20 \text { min nesta } \\
\text { etapa, realizando } 2-3 \text { séries e } 8-10 \text { repetições. Exercícios } \\
\text { de baixa carga/ bandas elásticas de média resistência. Por } \\
\text { alguns períodos são realizados exercícios com protocolos } \\
\text { de hidroterapia, como objetivo o ganho de resistência } \\
\text { cardiovascular. }\end{array}$ & $\begin{array}{l}\text { Exercícios de resistência realizando caminhada } \\
\text { na esteira em baixa velocidade e bicicleta } \\
\text { ergométrica com carga moderada com duração } \\
\text { de } 30 \text { min. }\end{array}$ \\
\hline C & $\begin{array}{l}\text { Exercícios de fortalecimento e resistência na água, priorizando } \\
\text { grandes grupos musculares dos MMII/MMSS e de tronco } \\
\text { utilizando flutuadores e halteres tendo duração de } 40 \text { min e } \\
\text { todos os exercícios são realizados em forma de protocolos. }\end{array}$ & $\begin{array}{l}\text { Exercícios ritmados baseados em FC durante } \\
\text { um período pré-determinado, e interrompidos } \\
\text { conforme os pacientes se aproximem da FC alvo } \\
\text { do treinamento de resistência. }\end{array}$ \\
\hline D & $\begin{array}{l}\text { Exercícios de fortalecimento e tônus muscular de grandes } \\
\text { grupos musculares de MMSS e MMII, utilizando halteres e } \\
\text { caneleiras com } 3 \text { séries e } 10 \text { repetições durante } 10 \text { min. }\end{array}$ & $\begin{array}{l}\text { Exercício de resistência cardiovascular, paciente } \\
\text { fica na esteira/bike durante } 30 \text { min com velocidade } \\
\text { moderada e treinamento baseado na FC alvo. }\end{array}$ \\
\hline
\end{tabular}

Legenda: MMSS - membros superiores; MMII - membros inferiores; FC - frequência cardíaca. 
Tabela 5 - Protocolos fisioterapêuticos dos programas de reabilitação cardíaca acerca do desaquecimento e relaxamento.

\begin{tabular}{ll}
\hline Serviços & Desaquecimento/Relaxamento \\
\hline A & Alongamento e relaxamento de 10 a 15 min \\
B & Alongamentos de grandes grupos musculares de MMSS e MMII de 10 a 15 min \\
C & São realizados relaxamento e desaquecimento por 20 min \\
D & Alongamentos de grandes grupos musculares e verificação completa de sinais vitais \\
\hline
\end{tabular}

Legenda: MIMSS - membros superiores; MIIII - membros inferiores.

\section{DISCUSSÃO}

No presente estudo foi realizada uma investigação epidemiológica acerca dos programas de reabilitação cardíaca na região central do estado do Rio Grande do Sul. Nesta pesquisa foram coletados dados em relação à prevalência das doenças cardiovasculares nos pacientes dos serviços de RC, as faixas etárias, os tipos de exercícios e a frequência semanal, a equipe multidisciplinar e a estrutura do local.

Segundo a Associação Americana do Coração, a Associação Americana de Reabilitação Cardiopulmonar e o Colégio Americano de Cardiologia (AHA/AACVPR/ ACC), os componentes de um programa de reabilitação cardiovascular devem abranger na avaliação do paciente: a história clínica do paciente, o exame físico, os exames auxiliares, o eletrocardiograma (ECG), os testes de exercícios(teste cardiopulmonar e ergometria), o teste de caminhada de seis minutos, as análises de laboratórios e outros, o diagnóstico de depressão e sobre a qualidade de vida dos pacientes. ${ }^{11}$ Todos os serviços estão de acordo com a última diretriz vigente, porém os serviços como $A, B$, e $D$ trazem uma diferença em não utilizar questionários de qualidade de vida e da depressão. Existem registros de alguns estudos que os pacientes em $\mathrm{RC}$, além de apresentarem uma melhora significativa da parte física e funcional, também tiveram uma melhora importante na emocional e psicológica, e essa melhora foi mantida até seis meses, após o programa; o que justifica a utilização de profissionais da área da saúde mental e a aplicação do questionário de qualidade de vida como de fato foi feito pelo serviço $C^{12,13}$

Os protocolos de atendimento são compostos basicamente de três elementos: treinamento físico supervisionado de acordo com prescrições individuais, reeducação do paciente e orientação para este e seus familiares. A prescrição de exercícios só é feita após uma avaliação criteriosa de cada paciente, e deve ser realizada com base na $\mathrm{FC}$ ou equivalente ao consumo máximo de oxigênio $\left(\mathrm{VO}_{2}\right.$ pico) de pico, baseado no resultado do teste ergoespirométrico. ${ }^{14,15}$ Todos os serviços visitados apresentavam os três elementos que acabamos de citar que devem fazer parte do programa de RC. Também a diretriz recomenda que os mesmos devam ser observados de forma contínua, o serviço $D$, ao qual temos como referência no Brasil e na América Latina, também contempla os itens relevantes aos elementos básicos presentes.

Em relação à frequência e a duração dos programas, o que é recomendado no geral são três a cinco vezes por semana, entretanto a maior parte dos nossos serviços era oferecido duas vezes por semana. ${ }^{16,17}$ Com relação ao tempo de cada sessão, os serviços pesquisados ficavam mais tempo com seus pacientes, pois realizavam monitoramento severo de sinais vitais e também dados de pesquisas eram coletados. Quanto à monitoração de sinais vitais nos serviços avaliados segue a recomendação da diretriz bem como o modelo do serviço $D$ de referência. Isto demonstra uma grande preocupação com a segurança dos pacientes bem como o sucesso do mesmo.

Todo programa de RC deve conter uma equipe multidisciplinar composta por um médico cardiologista, ou médico do exercício habilitado, enfermeira, um especialista em exercício (educador físico ou fisioterapeuta), nutricionista, psicológico, e uma assistente social. Esses profissionais são importantes porque visam oferecer ao paciente atendimento e educação completa, com experiência no controle de fatores de riscos cardiovasculares, a avaliação e intervenção no aspecto psicossocial e modificação de suas condutas e no estilo de vida. ${ }^{11}$ Sabendo da dificuldade financeira de manter uma equipe completa para o atendimento multidisciplinar no paciente com doença cardiovascular, todos os serviços do estudo tentavam manter a equipe com maior número de profissionais da área da saúde, para tentar dar assistência integral ao paciente. No estudo em questão, os serviços $A, B$ e $D$ são constituídos por uma equipe completa, diferente de $\mathrm{C}$, demonstrando um ponto frágil relacionada a essa questão.

Durante o atendimento em um serviço de reabilitação cardíaca é importante ter uma supervisão médica especialista em RCV na gestão, caso possa ocorrer complicações. Quando houver ausência de tal especialista, é indicado no local ter pessoas capacitadas na reanimação cardiovascular de emergência. ${ }^{18,19}$ Nos serviços pesquisados, três serviços possuíam uma supervisão médica adequada, durante os atendimentos, porém no serviço $C$ não, mas ao invés disso havia um profissional capacitado caso ocorresse algum determinado evento cardíaco, como a última diretriz vigente recomenda.

$O$ aquecimento engloba alongamentos de pequenos e depois grandes grupos musculares, com exercícios de forma lenta para posteriormente, aumentar essas repetições em menor tempo. Depois de cinco minutos é realizado um aquecimento mais intenso, incluindo trote ou algum exercício de maior intensidade, conforme a última diretriz. ${ }^{11,20-22}$ Por outro lado, Regenga ${ }^{23}$ prefere utilizar exercícios de alongamentos dinâmicos, aeróbicos e de coordenação associados a exercícios respiratórios, com duração de 5 à 10 minutos, para assim, preparar os sistemas cardiorrespiratórios e musculoesqueléticas para a continuação do protocolo de exercício específico. Todos os serviços pesquisados estão de acordo com os últimos autores citados.

Durante o condicionamento, o programa engloba os exercícios de fortalecimento, os exercícios aeróbicos e de resistência muscular. A Diretriz Sul-Americana de 
Prevenção e Reabilitação Cardiovascular prefere dividir essa fase em exercícios de resistência e aeróbicos. ${ }^{1} \mathrm{~A}$ realização de exercício aeróbico e sua intensidade devem se ajustar ao quadro clínico, estratificação de risco e aos objetivos do paciente. Deve-se utilizar 70-90\% da FC máxima alcançada, ou entre 50 a $80 \%$ da FC de reserva. A duração deve ser no início de trinta minutos, progredindo até uma hora de exercício continuo ou intermitente e as sessões podem ser de duas a cinco vezes por semana. ${ }^{11,24-27}$ Os indivíduos podem fazer o uso, nesta etapa do cicloergômetro, esteira rolante, bicicleta e de caminhadas, começando com quinze minutos iniciais, e posteriormente progredindo o tempo. Os serviços A, B, C e D estão em conformidade com a última diretriz vigente, demonstrando o quanto eles realizam este protocolo com grande eficácia.

Durante os exercícios de resistência ou exercícios resistidos, o fortalecimento muscular deve começar com cargas leves e progredindo ao longo das sessões. Esta atividade pode ser realizada de duas a três vezes por semana, com repetições de 8 a 15 por grupo muscular, a intervalos de trinta segundos a um minuto, com duração de quarenta minutos. Podendo ser utilizados pesos livres ou musculação, e pilates com práticas de resistência com flexibilidade e respiração.11,12,30 Afirmando o mesmo que os autore ${ }^{14,24,28}$ concordam com esse tipo de condutas realizadas durante essa etapa, porém ressalta que esta modalidade não pode ser em dias consecutivos.

Durante a última etapa chamada de desaquecimento ou relaxamento, os alongamentos devem ser realizados progressivamente, sendo praticados no começo ou no final das sessões de reabilitação. Algumas práticas como loga e Tai Chu Chuan podem auxiliar na redução da pressão arterial e no consumo máximo de $\mathrm{O}_{2} \cdot{ }^{20,29,30}$ Os serviços $\mathrm{A}, \mathrm{B}, \mathrm{C}$, e D estão de acordo com os autores previamente citados, porém nenhum deles utiliza as práticas inovadoras.

Segundo revisão sistemática e meta-análise, ${ }^{31}$ o treinamento físico baseado nas reabilitações de indivíduos cardiopatas em hidroterapia tem demonstrado uma grande melhora em relação aos sintomas, a capacidade de exercício e na qualidade de vida, com o impacto menor sobre as readmissões hospitalares e na mortalidade. Portanto, o serviço C que realiza o programa de reabilitação cardíaca totalmente em hidroterapia, pode trazer grandes benefícios aos seus pacientes e principalmente relaxamento ao final das suas sessões.

\section{CONSIDERAÇÕES FINAIS}

Foi possível perceber com essa pesquisa o quão complexo é montar um serviço de reabilitação cardíaca adequado para os pacientes cardiopatas, pois cada um depende de uma boa estrutura do local, os equipamentos, as pessoas especializadas e capacitadas na área de cardiologia. Um fator de extrema importância, durante os aspectos práticos da RC, é o monitoramento durante todo o decorrer do atendimento, pois já temos dados descritos na literatura que a cada 60.000 a 80.000 horas de reabilitação vai acontecer um óbito mesmo com toda equipe completa presente no local, e com desfibrilador automático disponível.

Por fim, de acordo com os dados que coletamos a respeito dos serviços de RC de Santa Maria - RS comparado a um hospital de referência e a diretriz atual, observamos que, na prática, se aproximam muito das recomendações descritas na literatura vigente. O que destacamos como um ponto positivo para uma cidade de médio porte como Santa Maria e que tem uma rede de saúde com alicerce acadêmico.

Não encontramos na literatura, estudos sobre o número ou distribuição de serviços de RC no Brasil. Assim, vemos ainda a necessidade de expandir estudos como esse para todas as regiões, para podermos definir melhor o perfil dos serviços de reabilitação cardíaca. Estamos cientes que este trabalho deve ser expandido para todo o Brasil; já estamos trabalhando em projetos futuros para continuar com esta pesquisa e com mais parceiros para que esta tarefa seja possível.

\section{REFERÊNCIAS}

1. Herdy $A H$, López-Jiménez $F$, Terzic $C P$, Milani $M$, Stein $R$, Carvalho T, Serra S, Araujo CG, Zeballos PC, Anchique CV, Burdiat G, González K, González G, Fernández R, Santibáñez C, Rodríguez-Escudero JP, Ilarraza-Lomelí H. Diretriz SulAmericana de Prevenção e Reabilitação Cardiovascular. Arq Bras Cardiol 2014;103(2Supl.1):1-31.

2. Brasil. Ministério da Saúde (MS) / Secretaria de Vigilância em Saúde (SVS) /Sistema de Informações sobre Mortalidade (SIM). Disponível em: http://tabnet.datasus. gov.br/cgi/deftohtm.exe?sim/cnv/obt10rs.def> Acesso em: 15/10/2015

3. Pashkow FJ, Dafoe WA. Clinical cardiac rehabilitation: a cardiologists' guide. 2nd ed. Baltimore: Williams \& Wilkins; 1999.

4. Moraes Ruy Silveira. Diretriz de reabilitação cardíaca. Arq Bras Cardiol 2005;84(5):431-40.

5. Silva E, Catai AP. Fase III. In: Pulz C, Guizilini S, Peres PA. Fisioterapia em cardiologia: aspectos práticos. São Paulo: Atheneu 2006;305-16.

6. Balady GJ, Fletcher BJ, Froelicher VF, Hartley LH, Krauss $\mathrm{RM}$, et al. Cardiac rehabilitation programs: a statement for healthcare professionals from the American Heart Association. Circulation 1994;(90):1602-10.

7. Pollock, ML, Schimidt DH. Doença cardíaca e reabilitação. 3.ed. Revinter: Rio de Janeiro, 2003.

8. American College of Sports Medicine. Diretrizes do ACSM para os testes de esforço e sua prescrição. 6.ed. Rio de Janeiro: Editora Guanabara Koogan S.A., 2003.

9. Brum PC, Forjaz CLM, Tinucci T, Negrão CE. Adaptações agudas e crônicas do exercício físico no sistema cardiovascular. Rev Paul Educ Fis, São Paulo 2004 Ago, 18:21-31.

10. Nunes RAM. Reabilitação cardíaca. São Paulo: Ícone, 2010.

11. AACVPR. Guidelines for Cardiac Rehabilitation and Secondary Prevention Programs. 4th ed. Champaign, IL: Human Kinetics Publishers, Inc; 2004.

12. Milani RV, Lavie CJ, Cassidy MM. Effects of cardiac rehabilitation and exercise training programs on depression in patients after major coronary events. Am Heart $J$ 1996;132(4):726-32.

13. Rossi RC, Vanderlei FM, Medina LAR, Pastre CM, Padovani CR, Vanderlei LCM. A influência do perfil clínico e sociodemográfico na qualidade de vida de cardiopatas submetidos à reabilitação cardíaca. ConScientia e Saude 2011;10(1):59-68. 
14. Taranto G, editores. Modificações na prescrição de exercício para pacientes cardíacos. In: Diretrizes do ACSM para os testes de esforços e sua prescrição. $7^{\mathrm{a}}$ Ed. Rio de Janeiro: Guanabara Koogan, 2007.

15. Araraki $\mathrm{H}$, Magalhães HM. Programas supervisionados em reabilitação cardiovascular - abordagem de prescrição de exercícios. Rev Soc Cardiol Estado de São Paulo 1996,6(1):23-30.

16. Steward KJ, McFarland LD, Weinhofer JJ, Cottrel E, Brown CS, Shapiro EP. Safety and efficacy of weight training soon after acute myocardial infarction. J Cardiopulm Rehabil 1998;18(1):37-44.

17. Dressendorfer RH, Franklin BA, Cameron JL, Trahan KJ, Gordon S, Timmis GC. Exercise training frequency in early postinfarction cardiac rehabilitation. Influence on aerobic conditioning. J Cardiopulm Rehabil 1995 JulAug:15(4):269-76.

18. Vongvanich P, Paul-Labrador MJ, Merz CN. Safety of medically supervised exercise in a cardiac rehabilitation center. Am J Cardiol 1996;77(15):1383-5.

19. Fletcher GF, Balady GJ, Amsterdam EA, Chaitman B, Eckel, $\mathrm{R}$, Fleg $\mathrm{J}$, et al. Exercise standards for testing and training: a statement for healthcare professionals from the American Heart Association. Circulation 2001;104(14):1694-740.

20. ACSM's. Guidelines for graded exercise testing and prescription. 6th ed. Philadelphia: Lippincott Williams \& Wilkins; 2000.

21. Brodie D, Bethell H, Breen S. Cardiac rehabilitation in England: a detailed national survey. Eur J Cardiovasc Prev Rehabil 2006;13(1):122-8.

22. Giannuzzi P, Mezzani A, Saner H, Björnstad H, Fioretti $P$, Mendes M, et al; Working Group on Cardiac Rehabilitation and Exercise Physiology. European Society of Cardiology. Physical activity for primary and secondary prevention. Position paper of the Working Group on Cardiac Rehabilitation and Exercise Physiology of the European Society of Cardiology. Eur $J$
Cardiovasc Prev Rehabil 2003;10(5):319-27.

23. Regenga, M. M. Reabilitação em Cardiologia: da unidade de terapia intensiva à reabilitação. São Paulo: Roca, 2000.

24. Pollock ML, Franklin BA, Balady GJ, Chaitman BL, Fleg JL, Fletcher, B, et al. AHA Science Advisory. Resistance exercise in individuals with and without cardiovascular disease: benefits, rationale, safety, and prescription: an advisory from the Committee on Exercise, Rehabilitation, and Prevention, Council on Clinical Cardiology, American Heart Association; Position paper endorsed by the American College of Sports Medicine. Circulation 2000;101(7):828-33.

25. Sociedade Brasileira de Cardiologia. [Guideline for cardiopulmonary and metabolic rehabilitation: practical aspects]. Arq Bras Cardiol 2006;86(1):74-82.

26. Seals DR, Hagberg JM, Hurley BF, Ehsani AA, Holloszy JO. Effects of endurance training on glucose tolerance and plasma lipid levels in older men and women. JAMA 1984;252(5):645-9.

27. Herdy $A H$, Zulianello RS, Antunes $M H$, Benetti M, Ribeiro JP. High intensity aerobic exercise training induces similar or even superior blood pressure reducing effects in controlled hypertensive patients. Eur Heart J. 2010;384(Suppl):Poster 2292.

28. Gonçalves ACCR, Pastre CM, Filho JCSC, Vanderlei LCM. Exercício resistido no cardiopata: revisão sistemática. Fisioter Mov 2012;25(1):195-205.

29. Patel C, North WR. Randomised controlled trial of yoga and bio-feedback in management of hypertension. Lancet 1975;2(7925):93-5.

30. Afilalo J, Karunananthan S, Eisenberg MJ, Alexander KP, Bergman $\mathrm{H}$. Role of frailty in patients with cardiovascular disease. Am J Cardiol 2009;103(11):1616-21.

31. Adsett JA, Mudge AM, Morris B, Kuys S, Paratz JD. Aquatic exercise training and stable heart failure: A systematic review and meta-analysis. Int J Cardiol 2015;186:22-28. doi: 10.1016/j.ijcard.2015.03.095

Como citar: CASTAGNA, Leticia; NARDI GOMES, Tiago José. Cenário da reabilitação cardíaca no centro do estado do Rio Grande do Sul. Cinergis, Santa Cruz do Sul, v. 18, n. 4, out. 2017. ISSN 2177-4005. Disponível em: <https://online.unisc.br/seer/index. php/cinergis/article/view/9682 >. Acesso em: O6 dez. 2017. doi:http://dx.doi.org/10.17058/cinergis.v18i4.9682. 